\title{
FOCAL SETS IN CERTAIN RIEMANNIAN MANIFOLDS
}

\author{
by J. W. BRUCE and D. J. HURLEY
}

(Received 15th December 1983)

\section{Introduction}

In recent years the geometry of generic submanifolds of Euclidean space has been the object of much study. Thom hinted in [7] that the focal set of such a submanifold could profitably be studied by using the family of distance squared functions on the submanifold from points of the ambient space. For a generic submanifold the focal set is the catastrophe or bifurcation set of this family. The key to obtaining results on the local structure of this focal set is a transversality theorem of Looijenga [5]; for an alternative exposition see [8].

In this paper we shall show how to extend these results to generic submanifolds of a larger class of spaces, namely the complete, simply connected Riemannian manifolds without conjugate points. (For example complete, simply connected Riemannian manifolds with non positive sectional curvature; see [6] p. 100).

The hypotheses are fairly natural: the condition that the ambient manifold has no conjugate points means that it has no focusing itself which might interfere with those of a submanifold. The hypothesis of simple connectivity can be explained by the following example. Take a closed curve with an inflexion in the plane and enclose it in a rectangle.

Identify opposite sides of the rectangle to obtain a flat torus; clearly the focal set of the closed curve will wind around the torus an infinite number of times. Indeed with a suitable choice of normal at the inflexion the focal set will be dense in the torus. There is no hope of any nice local structure for this focal set, even if we perturb the curve.

Below we first show that the family of distance squared functions on the above spaces play the same role as in the Euclidean case. We then prove the transversality result which we use to obtain normal forms for the local structure of the focal set of generic submanifolds.

Throughout we shall use the notation of [8] and [3] (except where the latter conflicts with the former of course!) The main tools used below come from singularity theory, and for this reason we have given a detailed proof of the one result (Proposition 1) concerning Riemannian geometry.

In what follows $H^{n}$ denotes a simply connected complete Riemannian manifold of dimension $n$ with no conjugate points. It is well known that for every point $x$ in such a space the exponential map $\exp _{x}: T_{x} H \rightarrow H$ is a diffeomorphism; see [6] p. 102. One easily checks that the distance between two points $x, y \in H$, say $d(x, y)$, is given by $d(x, y)=$ $\left\langle\exp _{x}^{-1}(y), \exp _{x}^{-1}(y)\right\rangle^{1 / 2}$ where $\langle$,$\rangle is the Riemannian inner product. The map d^{2}: H \times H \rightarrow \mathbb{R}$ defined by $d^{2}(x, y)=(d(x, y))^{2}$ defines a smooth family of functions $d_{y}^{2}: H \rightarrow \mathbb{R}$ on $H$, parameterised by $H$, where $d_{y}^{2}(x)=d^{2}(x, y)$. 
If $M$ is a submanifold of $H, N(M)$ the normal bundle of $M$ in $H$, we denote by $\exp (M): N(M) \rightarrow H$ the corresponding exponential map. The set of critical values of $\exp (M)$ is called the focal set of $M$. Intuitively this is the light caustic of the initial wavefront $M$ in the inhomogeneous media $H$. Our first result shows that the focal set of $M$ is the bifurcation set of the family $d^{2}: M \times H \rightarrow \mathbb{R}$ given by the restriction of $d^{2}$ to $M \times H$ (see [1]). (That is the focal set consists of those points $y \in H$ with $d_{y}^{2}: M \rightarrow \mathbb{R}$ having a non Morse singularity at some point $x \in M$.)

For the proof of our first proposition we shall need various results from [3]. We use the notation used there except that tangent spaces, to $M$ at $x$ say, are denoted by $T_{x} M$, and covariant differentiation along a curve by $\nabla / d t$.

Proposition 1.1. Let $N(M)$ denote the normal bundle of $M$ in $H$ and $\exp (M): N(M) \rightarrow H$ the exponential map. The point $y \in H$ is a critical value of $\exp (M)$ with critical point over $x \in M$ if and only $d_{y}^{2}: M \rightarrow \mathbb{R}$ has a degenerate critical point at $x \in M$.

To prove this result we need to introduce the idea of an $M$-Jacobi field.

Definition 1.2. ([3] p. 221). Let $\tau$ be a geodesic segment in $H$ with $\tau(1) \in M$ and $i(1)$ perpendicular to $T_{\tau(1)} M$. A Jacobi field $J$ on $\tau$ is an $M$-Jacobi field if

(i) $J$ is perpendicular to $\tau$;

(ii) $J(1) \in T_{\tau(1)} M$;

(iii) $J^{\prime}(1)-S_{\dot{i}(1)} J(1)$ is perpendicular to $T_{\tau(1)} M$, where $S_{\dot{\tau}(1)}$ is the symmetric transformation on $T_{\tau(1)} M$ corresponding to the second fundamental form of $i(1)$.

Proof of Proposition 1. Let $g: H \rightarrow \mathbb{R}$ be the function $g(x)=d_{y}^{2}(x)$, and let $I$ be an open interval containing 0 with $\alpha: I \rightarrow H$ a curve in $H$. We define a map $r: \mathbb{R} \times I \rightarrow H$ by $r(t, s)=\exp _{y}\left(t \exp _{y}^{-1}(\alpha(s))\right)$. Now

$$
g(\alpha(s))=\int_{0}^{1}\left\langle\frac{\partial r}{\partial t}, \frac{\partial r}{\partial t}\right\rangle(t, s) d t
$$

from which it follows that

$$
(g \circ \alpha)^{\prime}(s)=2\left\langle\alpha^{\prime}(s), \frac{\partial r}{\partial t}(1, s)\right\rangle
$$

Since $(g \circ \alpha)^{\prime}(s)$ also equals $\left\langle\alpha^{\prime}(s), \operatorname{grad} g(\alpha(s))\right\rangle$ clearly

$$
\operatorname{grad} g(\alpha(s))=2 \frac{\partial r}{\partial t}(1, s)
$$

If $\alpha$ is actually a smooth curve on $M$ it follows that $g \mid M$ has a critical point at $\alpha(0)=x \in M$ if and only if

$$
\frac{\partial r}{\partial t}(1,0)=\dot{\gamma}(1)
$$


is perpendicular to $T_{x} M$, where $\gamma(t)=r(t, 0)$ is the geodesic joining $y$ and $x$. In [3] p. 255 it is proved that $y=\gamma(0)$ is a critical value of $\exp (M)$ over $x=\gamma(1)$ if an only if there exists a non zero $M$-Jacobi field $J$ on $\gamma$ with $J(0)=0$. Note that such a field must have $J(1) \neq 0$ since otherwise $x$ would be a conjugate point of $y$, contradicting our hypothesis. So we have to show that such a field exists if and only if the restriction of $g$ to $M$ has a degenerate critical point at $x$. To do this we need to compute the Hessian of $g$ at $x$.

Let $v, w$ be vectors in $T_{x} M$, with $\tilde{w}$ a smooth vector field on $M$ extending $w$. The Hessian form $H_{g}(v, w)=v(\tilde{w}(g))=v\langle\operatorname{grad} g, \tilde{w}\rangle=\left\langle\nabla_{v} \operatorname{grad} g, w\right\rangle+\left\langle\operatorname{grad} g, \nabla_{v}(\tilde{w})\right\rangle$. If $\bar{\nabla}$ is covariant differentiation on $M$, with respect to the induced connexion, then $\nabla_{v}(\tilde{w})=\bar{\nabla}_{v}(\tilde{w})+T_{v}(\tilde{w})$ (see [3] p. 191). It easily follows that at $x$, $\left\langle\operatorname{grad} g, \nabla_{v}(\tilde{w})\right\rangle=$ $\left\langle\operatorname{grad} g, T_{v}(w)\right\rangle=-\left\langle S_{\operatorname{grad} g} v, w\right\rangle$. On the other hand if $\alpha$ is a curve on $M$ as above with $\alpha^{\prime}(0)=v$ then

$$
\nabla_{v}(\operatorname{grad} g)=2 \nabla_{v}\left(\frac{\partial r}{\partial t}(1, s)\right)=\nabla_{\operatorname{grad} g}\left(\frac{\partial r}{\partial s}(1, s)\right)=\nabla_{\operatorname{grad} g}\left(\alpha^{\prime}(s)\right)
$$

since $\nabla$ is symmetric; so $H_{g}(v, w)=\left\langle\nabla_{\operatorname{grad} g} v-S_{\operatorname{grad} g} v, w\right\rangle=2\left\langle\nabla_{\dot{\gamma}(1)} v-S_{\dot{\gamma}(1)} v, w\right\rangle$.

Now if $\alpha^{\prime}(0)=v \neq 0$ and $H_{g}(v, w)=0$ for all $w \in T_{x} M$ we claim that $J(t)=(\partial r / \partial s)(t, 0)$ is an $M$-Jacobi field along the geodesic $\gamma(t)$. By [3] p. $174 J$ is a Jacobi field; (ii) above is trivial, and (iii) follows from the expression above for $H_{g}$. For (i) we note that $\langle J(1), \dot{\gamma}(1)\rangle=0$ and

$$
\begin{aligned}
\frac{d}{d t} & \langle J(t), \dot{\gamma}(t)\rangle=\frac{d}{d t}\left\langle\frac{\partial r}{\partial s}(t, 0), \frac{\partial r}{\partial t}(t, 0)\right\rangle \\
& =\left\langle\frac{\nabla}{d t} \frac{\partial r}{\partial s}(t, 0), \frac{\partial r}{\partial t}(t, 0)\right\rangle=\left\langle\frac{\nabla}{d s} \frac{\partial r}{\partial t}(t, 0), \frac{\partial r}{\partial t}(t, 0)\right\rangle \\
& =\left.\frac{1}{2}\left(\frac{d}{d s}\left\langle\frac{\partial r}{\partial t}(t, s), \frac{\partial r}{\partial t}(t, s)\right\rangle\right)\right|_{s=0}
\end{aligned}
$$

But

$$
\left\|\frac{\partial r}{\partial t}(t, s)\right\|^{2}=\left\|\exp _{y}^{-1}(\alpha(s))\right\|^{2}=(g \circ \alpha)(s)
$$

and $\left(g \circ \alpha^{\prime}\right)(0)=0$ by hypothesis, so $\langle J(t), \dot{\gamma}(t)\rangle=0$ all $t$. Clearly $J(0)=0$ so $J$ is as required. Conversely if $J(t)$ is a non zero $M$-Jacobi field on $\gamma$ with $J(0)=0$ then $J(1)=v \in T_{x} M$ is a non zero vector and from the expression for $H_{g}$ above we see that $x$ is a degenerate critical point of $g$.

To obtain information on the local structure of the focal set we now prove the required transversity result. To avoid rather tedious topological arguments we shall suppose that the submanifold $M$ is compact. 
Theorem 3. Let $M$, a compact manifold, and $H$ be as above. Let $W$ be a submanifold of the multi-jet space ${ }_{r} J^{k}(M, \mathbb{R})$ which is invariant under addition of constants: so for any $\left(q_{1}, \ldots, q_{r}\right) \in W$ and $c \in \mathbb{R},\left(q_{1}+c, \ldots, q_{r}+c\right) \in W$. For a residual set of embeddings $i: M \rightarrow H$ in the space of embeddings $\operatorname{Emb}^{\infty}(M, H)$ the jet extension

$$
{ }_{r} j_{1}^{k} d^{2}: M^{(r)} x H \rightarrow{ }_{r} J^{k}(M, \mathbb{R})
$$

is transverse to $W$.

As usual in providing results of this type we need to find a suitable family of deformations of any smooth embedding $i: M \rightarrow H$. We shall use polynomial mappings $H \rightarrow H$ to deform $i$. Let $P_{k}$ denote the space of polynomial maps $\phi: \mathbb{R}^{n} \rightarrow \mathbb{R}^{n}$ of degree $k$. If $g: M, u_{0} \rightarrow \mathbb{R}^{n}, x_{0}$ is the germ of an embedding, $\phi_{1} \in P_{k}$ a polynomial map yielding the germ of a diffeomorphism $\phi_{1}: \mathbb{R}^{n}, x_{0} \rightarrow \mathbb{R}^{n}, y_{0}$ and $f: \mathbb{R}^{n}, y_{0} \rightarrow \mathbb{R}$ is the germ of a smooth function we define $F: M \times P_{k+1}, \quad\left(u_{0}, \phi_{1}\right) \rightarrow \mathbb{R}$ by $F(u, \phi)=f \circ \phi \circ g(u)$. Let $j_{1}^{k} F: M \times P_{k+1},\left(u_{0}, \phi_{1}\right) \rightarrow M \times J^{k}(m, 1)$ be the associated jet extension, where $m=\operatorname{dim} M$ (and we have chosen local co-ordinates on $M$ at $u_{0}$ ). They key step in the proof of Theorem 3 is

Lemma 4. (i) If $f$ is the germ of a submersion then $j_{1}^{k} F$ is the germ of a submersion. (ii) If $f$ has an $A_{1}(M o r s e)$ singularity at $x_{0}$ then the germ $j_{1}^{k} F$ is transverse to the manifold of constant jets.

Proof. Without loss of generality we may suppose that $M, u_{0}=\mathbb{R}^{m}, 0, x_{0}=y_{0}=0 \in \mathbb{R}^{n}$ (the constant part of $\phi_{1}$ is irrelevant). If $\psi: \mathbb{R}^{n}, 0 \rightarrow \mathbb{R}^{n}, 0$ is the germ of any diffeomorphism, one easily checks that the map $\phi \mapsto j^{k+1}\left(\psi^{-1} \circ \phi\right)$ is the germ of a diffeomorphism $P_{k+1}, \phi_{1} \rightarrow P_{k+1}, j^{k+1}\left(\psi^{-1} \circ \phi_{1}\right)=\phi_{2}$. Since the conclusions of both parts of the lemma only depend on the $(k+1)$-jet of $F$ it is enough to prove the lemma with $F$ replaced by $F_{1}: M \times P_{k+1},\left(0, \phi_{2}\right) \rightarrow \mathbb{R}$ where $F_{1}(u, \phi)=(f \circ \psi) \circ \phi \circ g(u)$ for any $\psi$ as above. Thus if the lemma holds for $f$ it holds for any germ right equivalent to $f$. Consequently we may suppose that in case (i) $f(x)=x_{1}$ and for (ii) $f(x)=\sum_{r=1}^{n} \varepsilon_{r} x_{r}^{2}, \varepsilon_{r}= \pm 1$.

Writing $e_{p}$ for the $p$ th unit vector in $\mathbb{R}^{n}$ consider the paths $s \mapsto \phi_{1}+s j^{k}\left(h \circ \phi_{1}\right) e_{p}$ in $P_{k+1}$ where $h(x)$ is a polynomial of degree $\leqq k$. The image of the tangent vector $\left(0, j^{k}\left(h \circ \phi_{1}\right) e_{p}\right)$ under the derivative of $j_{1}^{k} F$ is

$$
\lim _{s \rightarrow 0}\left(j^{k}\left(f \circ\left(\phi_{1}+s h \circ \phi_{1} e_{p}\right) \circ g(u)\right)-j^{k}\left(f \circ \phi_{1} \circ g\right)(u)\right) s^{-1}
$$

In case (i) this reduces to $\delta i p j^{k}\left(h \circ\left(\phi_{1} \circ g\right)(u)\right)$ and in case (ii) to $2 \varepsilon_{p} j^{k}\left(\left(\phi_{1} \circ g\right)_{p}(u) \cdot h \circ\left(\phi_{1} \circ g\right)(u)\right)$ where the second suffix $p$ denotes the $p$ th component of $\phi_{1} \circ g$. Using the fact that $\phi_{1} \circ g$ is an immersion, and $h$ can be chosen to be any polynomial of degree $\leqq k$ the result follows.

We can now give the

Proof of Theorem 3. Let $M \rightarrow H$ be an embedding. By identifying $H$ with $\mathbb{R}^{n}$ we find that $P_{k+1}$ gives a family of polynomial maps $H \rightarrow H$, and we consider the map $D^{2}: M \times H \times P_{k+1} \rightarrow \mathbb{R}$ defined by $D^{2}(u, y, \phi)=d^{2}(\phi(u), y)$, where $M$ is considered a 
submanifold of $H$. The map $d^{2}(-, y): H \rightarrow \mathbb{R}$ is smooth and has only one singular point, at $y$, which is easily seen to be an $A_{1}$ (a non degenerate minimum in fact). Now let $U$ be a bounded open neighbourhood of $M$ in $H$ and let $V$ be an open neighbourhood of Id, the identity map, in $P_{k+1}$ such that $\phi: U \rightarrow \phi(U)$ is a diffeomorphism for $\phi \in V$. Then Lemma 1.4 shows that the restriction $j_{1}^{k} D^{2}: M \times H \times U \rightarrow J^{k}(M, \mathbb{R})$ will be transverse to any submanifold $W$ of $J^{k}(M, \mathbb{R})$ invariant under addition of constants. Applying Thom's transversality lemma ([8] Section 2) now shows that for almost any $\phi \in U$, in the sense of Lebesgue measure, $j_{1}^{k} d^{2}: \phi(M) \times H \rightarrow J^{k}(M, \mathbb{R})$ will be transverse to $W$. The theorem now follows, in the case $r=1$, by the usual results (see [8]).

F or $r>1$, the case of multi-jets, one uses the standard observation (as in [8], Section 2) that deformations on disjoint compact sets can be chosen independently, using partitions of unity.

If $N_{r}(M)$ consists of those vectors in $N(M)$ of length $r>0$ then $\exp (M)\left(N_{r}(M)\right)$ is called a parallel distance $r$ from $M$. Intuitively it is a wavefront evolved from the initial wavefront $M$.

Corollary 5. For a residual set of embeddings of $M$ in $H$ the focal set of $M$ has generic Lagrange singularities. Fixing $r$ the parallel of $M$ distance $r$ from $M$ will, for $a$ residual set of embeddings of $M$ in $H$ have generic Legendre singularities.

Proof. The focal set of $M$ is the bifurcation set of family of functions $d^{2}: M \times H \rightarrow \mathbb{R}$. The parallel of $M$ is the discriminant set of the family of functions $F=d^{2}-r^{2}: M \times H \rightarrow \mathbb{R}$ (that is the set of $y \in H$ with $D F_{y}(x)=F_{y}(x)=0$ for some $\left.x \in M\right)$. The result now follows from Theorem 3 and [1], [5]. For the focal set we take the stratification of the multi-jet space ${ }_{r} J^{k}(M, \mathbb{R})$ constructed by Looijenga in [5] (see also [8]). This is certainly invariant under addition of constants, and the focal set will be that part of the induced stratification of $H$ obtained from non Morse jets.

For the parallel we need to consider the subset of Looijenga's canonical stratification of singular jets with zero constant. Unfortunately this is not invariant under addition of constants! However if $D F_{y}(x)=F_{y}(x)=0$ then $y \neq x$ and $d_{y}^{2}: H, x \rightarrow \mathbb{R}$ is the germ of a submersion. A careful analysis of the proof of Theorem 3 shows that if $W$ is a submanifold of the singular jets with zero constant then $j^{k} D^{2}$ is transverse to $W$, essentially because we need only apply Lemma 4 (i).

Remarks. (1) It follows from the classification of functions of low codimension that for $n \leqq 5$ (resp. $n \leqq 6$ ) a generic focal set (resp. parallel) is locally diffeomorphic to the union of bifurcation (resp. discriminant) varieties of Arnold's simple singularities in general position.

(2) It follows from Looijenga's topological stability theorem that for a residual set of embeddings of $M$ in $H$ the focal set is topologically stable i.e. small perturbations of $M$ yield homeomorphic focal sets.

(3) For the parallels we need to fix $r$ in advance since for certain values of $r$ the advancing wavefronts undergo catastrophic changes with non generic singularities appearing. Generic such changes have been classified by Arnol'd in [2] for low values of $n$. It would be interesting to know if they are generic for submanifolds of our spaces $H$. (They are generic for surfaces in Euclidean space [4]). 


\section{REFERENCES}

1. V. I. ArNol'D, Critical points of smooth functions, Vancouver Int. Congress of Math. (1974).

2. V. I. Arnol'D, Wavefront evolution and equivariant Morse lemma, Comm. Pure and Applied. Maths. 9 (1976), 557-582.

3. R. L. Bishop and R. J. Crittenden, Geometry of Manifolds (Academic Press, New York, 1964).

4. J. W. Bruce, Wavefronts and parallels in Euclidean space, Math. Proc. Camb. Phil. Soc. 93 (1983), 323-333.

5. E. J. N. Looujenga, Structural stability of smooth families of $\mathrm{C}^{\infty}$-functions (Doctoral thesis, Universiteit van Amsterdam, 1974).

6. J. W. Milnor, Morse Theory (Annals of Maths. Studies 51, Princeton University Press, 1963).

7. R. Tном, Structural Stability and Morphogenesis (W. A. Benjamin, Readin, Massachusetts, 1975).

8. C. T. C. WALL, Geometric properties of generic differentiable manifolds, Geometry and Topology (Vol. III Springer Lecture Notes in Mathematics No. 597, 1976), 707-774.

Department of Mathematics

UNIVERSITY OF NEWCASTLE UPON TYNE
Department of Mathematics University College, Corle, Eire 